\title{
Integrated Supply Chain Automation using Blockchain
}

\author{
Malleshappa T. Bhagawati, P. Venkumar, H.R. Patil, C.M. Javalagi
}

\begin{abstract}
A Blockchain is a shared ledger distributed across a business network. Blockchain is creating extraordinary opportunities for businesses to come together in new ways such as creating new values, optimize ecosystems and reduce risk. Using this Blockchain virtually anything of value can be tracked and traded, without requiring a central point of control.Blockchain is disrupting the supply chain industry the way they Technology are currently operating, in terms of overcoming issues with their operating layer, Document layer and Messaging layer. One of the objectives of the study was to identify the relevant blockchain use cases that can address the current pain points of various participants in the integrated supply chain process and improve the current operational and technical environment.

Supply chain costs can typically amount to $70 \%$ and holds the most levers for optimization. Gaining effective visibility and insights into the operations delivers significant and sustainable benefits. The assessment methodology focused around existing processes, technology \& supply chain participant roles to establish common workflows across different participants, understanding of client's pain points, operational inefficiencies and technology maturity. Post which came up with an "Industry Circle" to clearly articulate participants and their respective data exchange patterns to recommend optimizations.

Supply and replenishment patterns become long term strategies rather than short term operational tactics.

With Blockchain enabled supply chain, bring in value in terms of full visibility, transparency, reduced time, security in the entire network (for example: components can be tracked from their manufacturing to finished product) and with Smart Contracts, SLAs executed automatically.
\end{abstract}

Keywords: Supply Chain, Manufacturing, Blockchain, Hyper Ledger Fabric, Permissioned Business Network.

\section{I.INTRODUCTION}

A Blockchain is a shared ledger distributed across a business network. Blockchain is creating extraordinary opportunities for businesses to come together in new ways such as creating new values, optimize ecosystems and reduce risk. Using this Blockchain virtually anything of value can be tracked and traded, without requiring a central point of control.Blockchain Technology is disrupting the supply chain

Revised Manuscript Received on December 30, 2019.

* Correspondence Author

Malleshappa .T Bhagawati, Department of Mechanical Engineering, Kalasalingam University, Krishnankoil, Tamil Nadu, India. malleshbhagawati@gmail.com

P.Venkumar, Department of Mechanical Engineering, Kalasalingam University, Krishnankoil, Tamil Nadu, India. p.venkumar@klu.ac.in

H.R.Patil, Department of Mechanical Engineering, Basaweshwar Engineering College, Bagalkot, Karnataka, India. hr_patil@yahoo.co.in

C.M. Javalagi, Department of Industrial \& Production Engineering, Basaweshwar Engineering College, Bagalkot, Karnataka, India. cmjavalagi@gmail.com industry the way they are currently operating, in terms of overcoming issues with their operating layer, Document layer and Messaging layer.

As per research, following pitfalls are identified in a supply chain network, although data standards and protocols exist, they are fragmented along the supply chain. No single participant has full visibility into the shipment life cycle although some documents are digitized, from 30 to 200 documents are still been processed per shipment manually More error-prone and Duplication of messages.

Leading to more Invoice Disputes and High Transaction and Settlement Fees Dispute resolution for shipment delays and damaged or lost goods caused cargo losses of \$55B in 2018. For supply chain, Blockchain has immense opportunities by eliminating physical paper from the supply chain by digitizing end to end supply lanes, enabling the synchronous flows of physical goods with associated documents and messages in real time. Blockchain also creates a single secure event driven and document exchange cloud-based platform supporting the global supply chain. [1], [12]

The assessment analysis includes Permissioned Blockchain (Hyperledger Fabric) value proposition for building transparency and improving operational efficiency in the Integrated Supply Chain network.

During this study, visited medical products manufacturing plant to understand their current manufacturing process, relations with vendors, sub-contractors to assess their existing pain points in an integrated supply chain network. Based on the outcome following pain points are clearly articulated. Currently they have no visibility into the vendor inventory and faces challenges with accuracy of information provided in vendor documents.

Manufacturing unit also utilizes sub-contractors for Conversion/Machining. These sub-contractor's IT system lacks maturity and unable to feed required supply chain data into manufacturing systems. Transparency and traceability of movement of orders/assets between vendors and their suppliers is a major area concern for manufacturing plant. Manufacturing plant vendors many times falls shorts of inventors causing delays in fulfillment of order, causing delays [1],[3], [4], [6].

Blockchain Technology (Hyper Ledger Fabric 1.4) has the feature of immutability, traceability through consensus of all participants within the business network through smart contract execution \& crypto for secured transactions. 
In the context of a selected use case for a manufacturing industry, Blockchain helps to share data in real time with consensus from all participants involved. In this case manufacturing plant, assembly plant, suppliers and vendors share their current status of either transactions or documents related to supply chain events are all shared within the business network so that everyone has the visibility of supply chain and in turn building trust in the supply chain network. Existing issues of integrated supply chain can be resolved with centralized database, since database is centrally controlled and easily tampered without the notice of other participants in the network, Blockchain technology is selected due to its immense nature of distributed ledger secured through industry standard Crypto technology.

Transactions are committed to each of the participants ledger only after it get consensus through automatic execution of smart contracts at each of the organization (or peer) level, hence bringing in value from moving away from centrally controlled database technology to distributed ledger.

Manufacturing plant vendors many times falls shorts of inventors causing delays in fulfillment of order, causing delays.[1],[3], [4], [6]

\section{APPROACH AND METHODOLOGY ADOPTED}

The assessments discussions were carried out with around 20 participating entities and face to face discussions with 35 participants. the whole exercise spanned 14 sessions over 60 days [11]..A structured questionnaire was created and shared with the participants before the sessions. The questionnaire covered the following areas to gather required information:

Role in existing supply chain Process:- The existing supply chain includes Procurement(Bought out/ Manufactured/Fabricated), Planning(planning Lead Times, processing Lead Times), Production(Assembling, Testing and Validation) and Subcontracting.

End to End Business Process :- understanding of current supply chain process and activities

User Management:- How identity management is managed in the current IT systems. It is noted that through date base driven ID and roles are managed for SAP and few of the vendor systems.

System Architecture:-current system technical assessment is done to analyse currently integrated systems, security requirements for each of the systems as well kind of data maintained.

Compliance Requirements:-discussed with governance process and IT departments, to capture compliance

requirements related to technical, legal, and corporate requirements.

Infrastructure:- Assessed current IT infrastructure and found out the most of the systems are hosted on-prem on Nonstandard data centers without a disaster recovery in place. Participant's expectations from assessments

The assessment approach was focused around establishing common workflows across different participants, understanding of pain points, operational in-efficiencies (if any), and technology maturity.

The following steps were performed for the Current State Assessment phase:

Identification of key stakeholders- organizations and business units:- Multiple discussions carried out with ordering and manufacturing plant departments, vendors, sub-contractors and domestic transporters to understand their key pain points as an input to Blockchain solution value drivers

Planning of discovery workshops and deep dive sessions with identified stakeholders. These stakeholders will be considered for the Blockchain network participant roles- data input systems, regulatory/event monitoring nodes, transaction processing nodes (banking), user input screens and workflow/consensus roles.

Face to face sessions to understand current technology landscape, operational procedures and pain points.

Review various supply chain related documents- manual submissions and system uploads. Identify redundancies, data fraud scenarios and non-availability of critical information.

Discuss about the capabilities of a distributed ledger system to develop an international trade platform.

Once the common workflows and IT landscape was baselined, the current state was analysed using three lensesProcess, Technology and Network Participant Roles.

\section{A.Process Lens}

Following process evaluations carried out to arrive at Blockchain solution.

Internal Processes:- Processes that are internal to an organization, but the input or output values/results has direct or indirect impact on the supply chain process.

Ordering Plant:- Intercompany Pos ( sales order) process through SAP to its subsidiary manufacturing plant

Manufacturing plant:- PO acceptance, work flow, subcontracting work flow, QC process, labeling Kitting, Production assembly, Packing, logistics(Outbound)

Subcontractors/ Vendors:-Manufacturing process as per the design shared by the engineering team fro manufacturing unit to invoicing process.

Processes that are directly linked to the supply chain.- It includes creation and assignment to Vendors/ subcontractors, Vendors shipment process, carrier delivery process, warehouse Inbound and Outbound Process and Custom Process.

Processes that are manual in nature and need to be transformed/automated to a digital platform:-PO creation from assembly/manufacturing plant to it's vendors/sub-contractors, Inventory check of vendors/sub-contractors before PO issue, Vendors document exchanges. QC results Communication between different participants of this supply chain Logistics, document exchange and Customs document submission

Processes that have dependencies on multiple parties for completion:- PO \& Invoice, QC check, Domestic transport Customs clearance 
Processes that require faster turnaround time:- SLA adherence, Domestic transport, Customs clearance, Document exchange

Processes that are critical from compliance and regulation perspective:- $\mathrm{PO}, \mathrm{SO}, \mathrm{BOL}, \mathrm{AWB}$, Packaging List, Tax receipts from Customs clearance \& domestic transport perspective.

\section{B.Technology Lens}

Currently there are no data privacy requirements for data exchange; all supply chain documents exchange has company details rather than personal data of an individual.

SAP \& few of the vendors data is online. There are no clearly defined policies or procedures related to data purging. Applications across Manufacturing Industries and vendor companies are hosted on-premise. Disaster recovery for critical applications hosted at manufacturing industries are physically located in the same building as the primary databases. None of the documents are digitized. Based on multiple discussions with key stakeholders, including domestic logistics, it has been observed that, all payments transactions are through either cash or cheque.

\section{C.Network Participant Roles}

Role of Manufacturing Units :- Place PO, approve Invoices, payments to vendors/sub-contractors, quality check of components received, QC of finished product, maintain all shipment documents, act as a custom broker to get approvals from customs, knitting, labelling and packaging of finished products.

Role of vendors \& sub-contractors:-- Acknowledge PO, build components as per design, ship the finished components and raise a payment request through Invoice.

Role of Customs Authority:- - Review of customs declaration and inspection of goods

Role of Private Agencies:- - Support domestic transport of either components from vendors/sub-contractors or from assembly units finished products.

This methodology allows for an all-encompassing view of the current supply chain process and lays the foundation for a blockchain based solution to be able to address the shortcomings of the existing process from the perspective of all the three lenses.

\section{III.CURRENT SUPPLY CHAIN}

\section{A.Scenario of Existing Manufacturing Plant SCM}

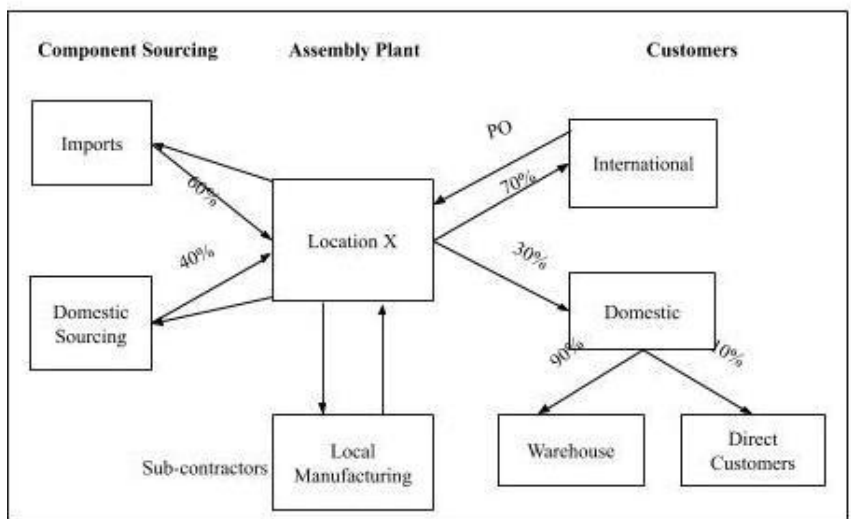

Figure 1: Manufacturing Industry Current Supply Chain Scenario

Manufacturing plant currently exports nearly $70 \%$ of the

Products manufactured, the balance is supplied to the local India market. During their manufacturing process, they have no visibility into the vendor inventory, due to which faces challenges with accuracy of information provided in vendor documents.

Unit also utilized sub-contractors for Conversion/Machining. These sub-contractors's IT system lacks maturity and unable to feed required supply chain data into manufacturing unit systems.

Transparency and traceability of movement of order/assets between vendors and their suppliers is a major area concern, vendors many times falls shorts of inventors causing delays in fulfillment of order, causing delays.

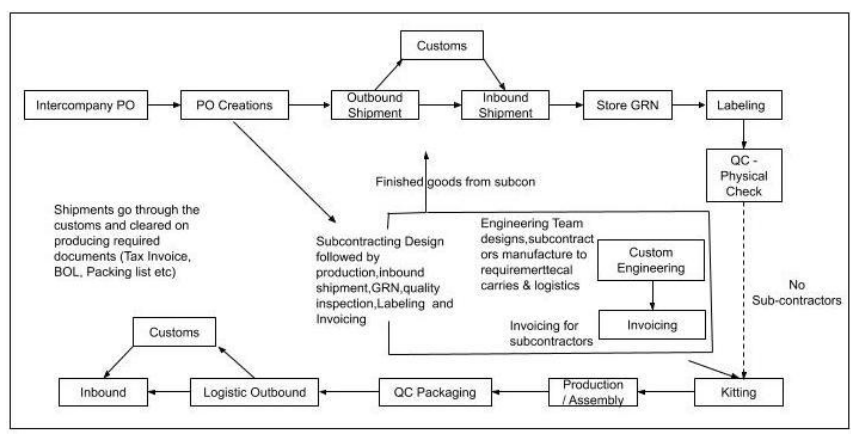

Figure 2: Manufacturing Industry Current SCM Process

Current process takes approximately around 7 weeks to 18 Weeks to complete impacting agreed SLA's with plants to deliver finished product.

\section{Existing Process and Activities}

Following is there existing process in an integrated-supply chain.

- Ordering plant creates Intercompany purchase order (PO) for its subsidiary manufacturing plant.

- PO created and assigned to vendor/subcontractor by purchase ops dept. PO is send through Email/EDI used.

- Vendor sends shipment with the physical documents (BoL, Item List, etc). Carrier raises a pre-alert \& shares DSR (Daily Shipment Report) with manufacturing unit Logistics dept.

- Carrier delivers shipment and physical documents to Unit. Documents include, GRN, Quality inspection report, Labelling \& Invoice.

- Unit depts. will store GRN, physical waybill signed to Carrier. Performs physical check for material received. QC creates MIGO for order in SAP.

- Labelling department labels the goods, QC documentation and specification check and finally puts a QC sticker.

- Kitting department do the kitting as per BOM and move material to the production area. 
- Production/Assembly unit assembles the product, Intimate QC for quality check. Unit also creates a packaging list and sends it to the logistics.

- Packaging department packs the assembled product and QC done, internal logistics team takes over product and documents.

- Logistics (Outbound) dept, finished product shipped with physical documents (BOL, Invoice, Tax, package list, etc.). Shipment goes through the customs and cleared on producing required documents (Tax Invoice, BoL, Packaging List, etc)

- In Bound department at ordering plant, once product reaches post customs clearance, ordering plant notifies for the carrier.

\section{TO-BE BUSINESS WORKFLOW WITH BLOCKCHAIN SOLUTION}

One of the objectives of the study was to identify the relevant blockchain use cases that can address the current pain points of various participants in the integrated supply chain process and improve the current operational and technical environment.

Currently the participants like ordering plant, manufacturing plant, vendor, sub-contractors, domestic transport, and outbound shipment departments have to engage in multiple channels- both manual and digital to exchange supply chain related documents. Considering the fact that, most of these documents are exchanged manually, the inherent redundancies, repetition and operational inefficiencies in the overall process is considerably high.

Blockchain can offer a single trade document exchange platform that will allow for single submission of documents, including bill of lading (BoL), purchase order, Invoices,

item list, QC, GRN, MIGO, Tax Invoice, etc. by the relevant network participant.

This will considerably reduce the time and effort taken to share these documents in a point to point exchange mechanism.

Documents will be digitized, and a single version of each document will be available to the concerned/relevant trade participant, only.

As depicted in below diagram, Following is the proposed Blockchain process for an integrated supply chain.

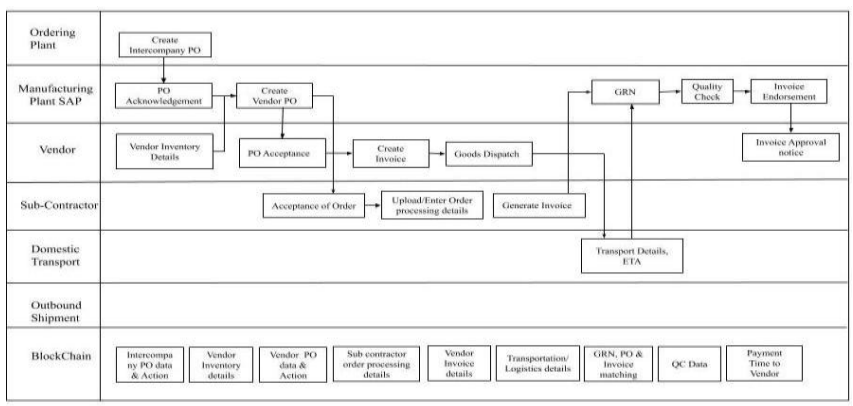

Figure 3: Optimized SCM Process

As soon as PO is raised from ordering plant, document will be stored on blockchain and event will be triggered to Manufacturing plant.

Once Manufacturer plant acknowledges the PO, smart contract on blockchain will be run to check the vendor inventory details as an Input for manufacturing plant before creating an purchase order with vendors. This will help manufacturing plant to assess the vendor inventory before raising the $\mathrm{PO}$ or give flexibility to go for some other vendor/sub-contractor on the network who has enough inventory to meet the product components demand.

Once PO is raised successfully by manufacturing plant, smart contract will trigger event to respective vendors/sub-contractors to take action. Once PO is accepted, Smart contract on blockchain will trigger a SLA measure counter so that agreed SLA between them will be met.

Vendor/sub-contractor ready with requested components, they will upload GRN, Invoice and goods dispatch note to Blockchain.

Goods dispatch note will trigger a smart contract to parse the document and trigger an action to Domestic transport particant so that he can take action to pick up goods and deliver to manufacturing unit.

Once goods delivered, Transport agency on Blockchain updates/upload relevant documents and action will be triggerred to manufacturing plant.

Manufacturing plant will perform their internal "Quality Check" process and upload the results to Blockchain.

Results of QC check will be updated to distributed ledger shared across all participants.

Once QC Check is successful, smart contract defined workflow will trigger action to manufactuing unit to approve Invoice and raise a payment trigger within the stipulated timelines.

Post QC check, Events will be triggerred to respective departments within manufacruting units for Kitting, delivery order generation, packaging list \& outbound dispatch. Participants on the blockchain network will have a fully visibility of each of the actions and progress on Blockchain.

\section{V.TECHNOLOGICAL COMPONENTS}

\section{A. System Context}

This Objective of this solution is to provide the transparency and traceability of movement of orders/assets between vendors and their suppliers for Manufacturing Industry through automation.

As depicted below in the system context,

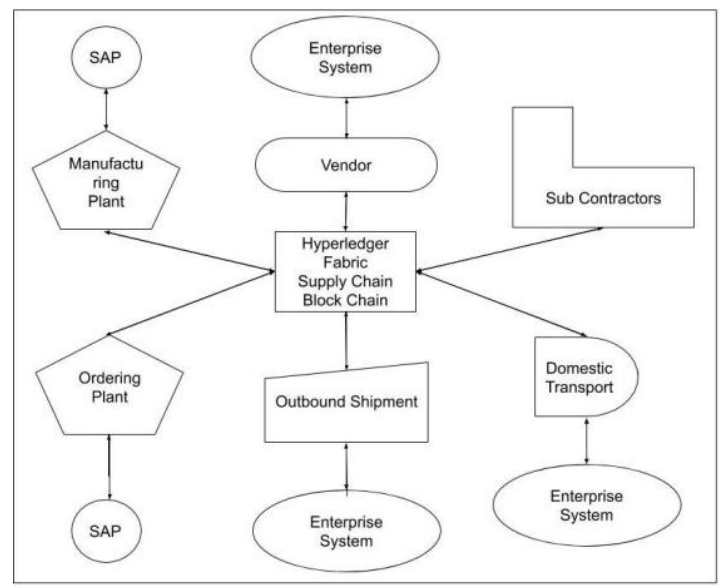

Figure 4 : Blockchain System Context 
Current integrated supply chain participants like Ordering plant, Manufacturing plant, Vendors, Sub-contractors, Outbound shipment and Domestic transport will share a common distributed ledger to get complete visibility of supply chain transactions.

Each of the participants IT systems like SAP (For Manufacturing plants \& Ordering Plants), Enterprise systems of vendors, outbound system and domestic transports are also integrated on blockchain through API integration so that data is fed continuously to distributed ledger.

The blockchain value drivers can be measured on two separate scales- network participants and internal processes. Network participants will benefit from the blockchain solution in terms of end to end visibility into the supply chain. They will have details related to the exact data and time for the arrival of the components, details related to the goods inbound, different quality inspections teams need to be involved, current capacity at the vendors/sub-contractors and single view into all supply chain related documents. The blockchain solution will also provide alerts and notifications to all concerned network members.

As the data to the blockchain network would be coming from source systems or permissioned blockchain network members, the confidence and trust in the system will vastly improve.

Any dispute on the blockchain network could be resolved using automated workflows and smart contracts. In case of a dispute, the relevant stakeholder will be notified and through corrective actions the dispute could be resolved, much faster. Operational efficiencies of various network members can also gain from an integrated blockchain solution. For example suppliers can leverage the real time purchase order and advance document availability on the blockchain network to optimize loading/unloading and movement of components, as well payment of invoices on time from manufacturers.

As blockchain will offer both workflow automation and smart contracts for rules processing, many manual activities, both across the network and internal, can be completed more efficiently and in less time. Resources tied up in data entry, dispute resolution, document management or reconciliation can be utilized in other areas to further improve internal processes and technologies.

Furthermore, a common SLA framework for the entire blockchain network can be established. As the blockchain platform will monitor all transactions and events on the network, the time taken to complete a specific request can be determined automatically. Like, time taken from PO submission to issue/ reject of payments of Invoices or the time elapsed for document submission or duty payments

\section{B.Blockchain Architecture Overview}

Proposed Architecture comprises of following solution Components.

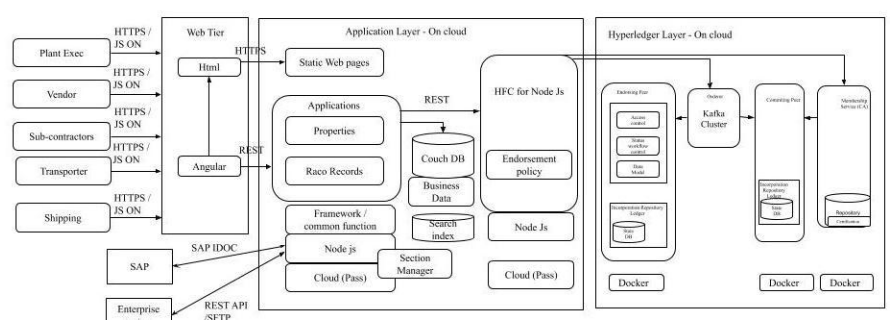

Figure 5: Blockchain SCM Architecture

\section{UI Components}

A Common Component Framework needs to be created to have commonly used sub-components to maintain in one place and shared across projects.

Three web Projects - one for each Organization (Plant, Vendor \& Transport)

Following are the user groups for each of the web applications Plant Web Application: Ordering Plant \& Manufacturing Plant

Vendor Web Application: Vendors, Sub-Contractors

TransportWebApplication:

Domestic

transporters, outbound shipment

Deploy it as three different projects with three different URLs to access it securely.

Client branding should be based on configuration (like Client Logo, Color code, etc.)

Integration Components:

Blockchain system will integrate with existing SAP systems of Ordering and Manufacturing/ Assembly Units through SFTP for exchange of PO \& Invoice details.

SOAP API integration with Assembly Units QC department system to capture the results of QC check for either at the Component level supplied by vendors or at the finished goods level.

Domestic transport systems expose their services through messaging gateway, hence data will be captured on blockchain through Rabbit MQ platform that will in turn call REST API's of smart contracts running on each peers of block chain network.

\section{Blockchain Components:-}

Following blockchain components help to maintain immutability within the supply chain business network for manufacturing unit.

Workflow Manager: Smart contract component to handle following workflows,

Purchase Order Flow: Workflow with events and documents meta data to notify next actor in the flow. Defined workflow will capture PO from Ordering plant, Acknowledgement from Assembly Unit, Shipment confirmation and Acknowledgement from ordering plant for successful delivery of finished product. This workflow also supports PO process between Assembly unit and vendors.

Inventory check and Approval flow:-This workflow mainly between Assembly unit and Vendors / sub-contractors to assess as of today inventory to support requested PO.Invoice Approval Flow: Automated process running on each peer's of blockchain network to check process steps before raising notification for approval of raised Invoices.

Quality Check Process: Workflow to raise an automated notification to QC departments and to capture the results of QC

Domestic Transport: Workflows:-Workflow to capture automated notifications to domestic transporters to take action once either components shipment is ready from vendors or finished good from assembly units.

This workflow also captures and make sure the defined document list is in place before raising the notification to transporters to avoid any delays in the integrated supply chain

Doc State Manager: It's a smart contract component that 


\section{Integrated Supply Chain Automation using Blockchain}

will make sure that documents are accessed securely and with relevant permissions.

Notification: Automated notification event from Blockchain for traceability purpose as soon as any of the data gets updated on blockchain ledger.

Shared Ledger: Each of Blockchain peer will maintain a distributed append-only ledger, which is internally managed by blockchain components to attain immutability.

A network of industry participants maintains a distributed, permissioned ledger with copies of document filings, relevant supply chain events, authority approval status, and full audit history; every change result in a new, immutable block [6], [7], [8], [9], [10] .

\section{VI.EXPECTED BENEFITS OUT OF BLOCK CHAIN SOLUTION}

\author{
A.SLA Adherence \\ Due to a common Blockchain collaboration platform, the \\ Production unit can meet the following SLA's, \\ According to Product Category \\ "A" Cat (High) - 4 weeks lead time \\ "B" Cat (Medium) - 6 weeks lead time \\ "C" Cat (Low) - 16 weeks lead time
}

\section{B.Platform Benefits}

Traceability for products in Logistics / Transit. Visibility into Supplier inventory

Quick customs clearance, reducing warehouse costing at Customs

All Documents will be stored securely on blockchain platform; this preserves authenticity and prevents fraud Sub-contractors are involved in the Blockchain platform, hence there is no need for each of them to develop their own systems / processes

\section{CONCLUSION}

This assessment for a manufacturing industry shows that the current documentation and tracking process is manual and overly complex, with blockchain technology introduction they can easily do,

Time tracking and monitoring of shipments at piece level on blockchain

Improve document sharing throughout the supply chain in the blockchain

Security throughout the supply chain

For all manufacturing industries to drive value with blockchain, the following approach has been suggested:

Dream big, act step by step,

Identify business challenges and opportunities,

Work together to create business value right from the start

Govern for the future.

Blockchain is a team sport - make connections!

\section{FUTURE WORK}

In this work, we have tried to optimize the existing operational processes of a Production unit using blockchain technology. However, this work can be easily extended to form a consortium of such diverse manufacturing organizations that come together and share data on a single platform. This will have a significant impact on overall supply chain network
AI: Artificial Intelligence

\section{NOMENCLATURE}

IP: Internet Protocol

IT: Information Technology

SLA: Service Level Agreement

PO: Purchase Order

EDI: Electronic Data Interchange

BOL: Bill Of Lading

DSR: Daily Shipment Report

GRN: Goods Received Report

QC: Quality Check

IOT: Internet Of Things

MIGO: Movement in Goods

SAP: Systems Applications \& Products

BOM: Bill of Materials

API: Application Program Interface

UI: User Interface

URL : Uniform Resource Locator

SFTP: Secure File Transfer Protocol

SOAP: Simple Object Access Protocol

\section{ACKNOWLEDGMENT}

We thank the team, the managers and the account leaders of the project experience shared - Shaji K Kamath and Sanjiv Gupta. Special thanks to ReenavMajumdar and Christian Stavek, technical teams who helped with the model design and owned explaining the functionality to the manufacturing organization. Our deepest gratitude to Raghu Rajan for supporting and making sure that all key stakeholders present and share their pain points during assessments

\section{REFERENCES}

1. How Blockchain Delivers Value in Manufacturing, IEEE Innovation

2. Smartsupply: Smart Contract Based Validation for Supply Chain Blockchain, IEEE, DOI:10.1109/Cybermatics_2018.2018.00186

3. Columbus, Louis. (28 Oct 2018). "How Blockchain Can Improve Manufacturing In 2019”. Forbes.

4. Continuous interconnected supply chain Using Blockchain \& Internet-of-Things in supply chain traceability, Deloitte

5. Paul Brody, "How Blockchain is revolutionizing supply chain”, EY Global Innovation Blockchain Leader

6. A Permissioned Blockchain Framework for Supporting Instant Transaction and Dynamic Block Size,2016 IEEE Trustcom/BigDataSE/ISPA

7. Zero to Blockchain, IBM Redbooks course

8. HyperledgerFabric,readthedocs,https://hyperledger-fabric.readthedocs io/en/release-1.4/blockchain.html

9. https://github.com/Kunstmaan/hyperledger-fabric-kuma-token-examp $\underline{\text { le }}$

10. https://github.com/yeasy/docker-compose-files/issues/51

11.,https://medium.com/patara/design-thinking-for-blockchains-ded1d6 cabe53

12. https://public.dhe.ibm.com/common/ssi/ecm/wh/en/whw12345usen/ watson-customer-engagement-watson-supply-chain-wh-white-paper-e xternal-whw12345usen-20180411.pdf 


\section{AUTHORS PROFILE}

Malleshappa T. Bhagawati is working as an Associate Prof. in Department of Production Engineering, Konkan Gyanpeeth College of Engineering Karjat, Maharashtra state, India. He holds B.E. in Industrial \& Production Engineering from Karnataka University Dharwad, Karnataka, India., M.Tech. Specializing in Production Management from Visveswarya Technological University Belagavi, Karnataka State, India. Also he had completed MBA specializing in Marketing Management from YCMOU University Nashik, Maharashtra State, India. $\mathrm{He}$ is a Senior Member of Indian Institute of Industrial Engineering. And Associate Member of Indian Institute of Materials Management. Currently he is pursuing his Ph.D. in Green Supply Chain Management from Kalasalingam Academy of Research and Education, Krishnankovil, Tamil Nadu, India. His areas of interest include Industrial Engineering, Green Supply Chain Management, Production \& Operations Management.

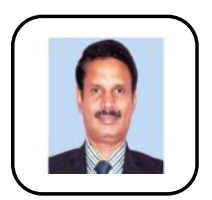

Dr. P. Venkumar, is currently a Senior Professor in Department of Mechanical Engineering, Kalasalingam Academy of Research and Education, Krishnankovil, Tamilnadu, India. He received his B.E., and M.E., degrees from Thiagarajar College of Engineering, Madurai, Tamilnadu, India, in 1994 and 1997, respectively and Ph.D. degree from Manonmaniam Sundaranar University, India in Industrial Engineering in 2006. His main areas of research interest are Manufacturing System Optimization, Supply Chain Management and Smart Manufacturing.

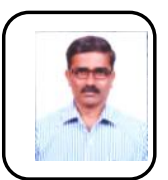

H. R. Patil is working as an Associate Professor in the Department of Mechanical Engineering, Basaveshwar Engineering College (Autonomous) Bagalkot, Karnataka, India. He has received his B. E degree in Industrial and Production Engineering from Karnataka University Dharwad, Karnataka, India. and M. Tech. in Production Technology from Visvesvaraya Technological University, Belagavi. Karnataka, India. Presently he is pursuing his research in Green Supply Chain Management. from Visvesvaraya Technological University, Belagavi, Karnataka, India. He is life member of ISTE.

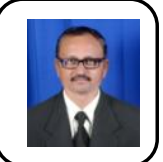

Dr. C. M. Javalagi received his B.E. degree in Mechanical Engineering and M.E. degree in Production Management from Karnataka University, Dharwad, Karnataka, India. in 1990, and 1992 respectively. Has completed Ph.D., from Visvesvaraya Technological University, Belagavi, Karnataka, India. in the year 2011. He is working as Professor in the Department of Industrial \& Production Engineering at Basaveshwar Engineering College (Autonomous) Bagalkot, Karnataka, India. He is life member of ISTE and Institution of Engineers. He has published many research papers in journals \& conferences at national and international level. He has more than 25 years teaching experience. His research interest includes Production Management, Supply Chain Management, and Operations Management. 